\title{
SOME NEW FORMULAS ON THE K-FIBONACCI NUMBERS
}

\author{
Sergio Falcon \\ Depatment of Mathematics \\ University of Las Palmas de Gran Canaria (Spain) \\ sergio.falcon@ulpgc.es
}

\section{ABSTRACT}

In this paper, we find some formulas for finding some special sums of the k-Fibonacci or the k-Lucas numbers. We find also some formulas that relate the k-Fibonacci or the k--Lucas numbers to some sums of these numbers.

\section{Keywords}

k-Fibonacci numbers, k-Lucas numbers, Binomial transform, Geometric sum.

\section{SUBJECT CLASSIFICATION}

MSC2000: 15A36; 11C20; 11B39.

\section{INTRODUCTION}

There exist generalizations of the classical Fibonacci numbers given by many researchers as Horadam [4] and recently by Falcon and Plaza[3].

\subsection{Definition of the k-Fibonacci numbers}

For any positive real number k, the k-Fibonacci sequence, say $F_{k}=\left\{F_{k, n}\right\}_{n \in N}$, is defined by the recurrence relation

$$
F_{k, n+1}=k F_{k, n}+F_{k, n-1}
$$

with initial conditions $F_{k, 0}=0, F_{k, 1}=1$.

For $k=1$, classical Fibonacci sequence is obtained and for $k=2$, Pell sequence appears.

We define the negative k-Fibonacci numbers as $F_{k,-n}=(-1)^{n+1} F_{k, n}$

In similar formFalcon [2], the k-Lucas numbers are defined as $L_{k, n+1}=k L_{k, n}+L_{k, n-1}$ with initial conditions

$L_{k, 0}=2, L_{k, 1}=k$.

The well-known Binet formula in the Fibonacci numbers theory $[5,3,1]$ allows us to express the k-Fibonacci and the kLucas numbers by mean of the roots $\sigma_{1}$ and $\sigma_{2}$ of the characteristic equation associated to the recurrence relation

$r^{2}-k \cdot r-1=0$. If $\sigma_{1,2}=\frac{k \pm \sqrt{k+4}}{2}$, then

$$
\begin{aligned}
F_{k, n} & =\frac{\sigma_{1}^{n}-\sigma_{2}^{n}}{\sigma_{1}-\sigma_{2}} \\
L_{k, n} & =\sigma_{1}^{n}+\sigma_{2}^{n}
\end{aligned}
$$

As a result of the above, $L_{k, n}=F_{k, n-1}+F_{k, n+1}$.

An interesting formula that we will apply next is the Convolution formula:

$F_{k, n+m}=F_{k, n+1} F_{k, m}+F_{k, n} F_{k, m-1}$.

Some properties of $\sigma_{1}$ and $\sigma_{2}$ that we will use in this paper are the following:

$\sigma_{1}+\sigma_{2}=k, \sigma_{1}-\sigma_{2}=k^{2}+4, \sigma_{1} \cdot \sigma_{2}=-1, \sigma^{2}=k \sigma+1, k-\sigma_{1}=-\frac{1}{\sigma_{1}}=\sigma_{2}$

\section{SOME NEW FORMULAS}

First we will find a formula that we will use for obtain other simpler formulas.

\subsection{Theorem}

For $a, r, p \in R$

$$
\sum_{j=0}^{n} \frac{F_{k, a j+r}}{p^{j}}=\frac{1}{p L_{k, a}-(-1)^{a}-p^{2}}\left[\frac{1}{p^{n}}\left(p F_{k, a(n+1)+r}-(-1)^{a} F_{k, a n+r}\right)+(-1)^{a} p F_{k, r-a}-p^{2} F_{k, r}\right]
$$

Proof. 


$$
\begin{aligned}
& \sum_{j=0}^{n} \frac{F_{k, a j+r}}{p^{j}}=\frac{1}{\sigma_{1}-\sigma_{2}} \sum_{j=0}^{n}\left(\frac{\sigma_{1}^{a j+r}}{p^{j}}-\frac{\sigma_{2}^{a j+r}}{p^{j}}\right)=\frac{1}{\sigma_{1}-\sigma_{2}}\left[\sigma_{1}^{r} \sum_{j=0}^{n}\left(\frac{\sigma_{1}^{a}}{p}\right)^{j}-\sigma_{2}^{r} \sum_{j=0}^{n}\left(\frac{\sigma_{2}^{a}}{p}\right)^{j}\right] \\
= & \left.\left.\frac{1}{\sigma_{1}-\sigma_{2}}\left[\sigma_{1}^{r} \frac{\left(\frac{\sigma_{1}^{a}}{p}\right)^{n+1}-1}{\frac{\sigma_{1}^{a}}{p}-1}-\sigma_{2}^{r} \frac{-1}{p}\right]=\frac{1}{\sigma_{2}^{a}} \frac{1}{p}-1\right] \sigma_{1}^{r} \frac{\sigma_{1}^{a(n+1)}-p^{n+1}}{\sigma_{1}^{a}-p}-\sigma_{2}^{r} \frac{\sigma_{2}^{a(n+1)}-p^{n+1}}{\sigma_{2}^{a}-p}\right] \\
= & \left.\frac{1}{p^{n}} \frac{1}{(-1)^{a}-p\left(\sigma_{1}^{a}+\sigma_{2}^{a}\right)+p^{2}} \frac{1}{\sigma_{1}-\sigma_{2}}\right] \\
& \cdot\left[\sigma_{1}^{r}\left((-1)^{a} \sigma_{1}^{a n}-p \sigma_{1}^{a(n+1)}-p^{n+1} \sigma_{2}^{a}+p^{n+2}\right)-\sigma_{2}^{r}\left((-1)^{a} \sigma_{2}^{a n}-p \sigma_{2}^{a(n+1)}-p^{n+1} \sigma_{1}^{a}+p^{n+2}\right)\right] \\
= & \frac{1}{p^{n}} \frac{1}{(-1)^{a}-p L_{k, a}+p^{2}} \frac{1}{\sigma_{1}-\sigma_{2}} \\
& \cdot\left[(-1)^{a}\left(\sigma_{1}^{a n+r}-\sigma_{2}^{a n+r}\right)-p\left(\sigma_{1}^{a(n+1)+r}-\sigma_{2}^{a(n+1)+r}\right)-p^{n+1}(-1)^{a}\left(\sigma_{1}^{r-a}-\sigma_{2}^{r-a}\right)+p^{n+2}\left(\sigma_{1}^{r}-\sigma_{2}^{r}\right)\right] \\
= & \frac{1}{p^{n}} \frac{1}{(-1)^{a}-p L_{k, a}+p^{2}}\left[(-1)^{a} F_{k, a n+r}-p F_{k, a(n+1)+r}-(-1)^{a} p^{n+1} F_{k, r-a}+p^{n+2} F_{k, r}\right] \\
= & \frac{1}{p L_{k, a}-(-1)^{a}-p^{2}}\left[\frac{1}{p^{n}}\left(p F_{k, a(n+1)+r}-(-1)^{a} F_{k, a n+r}\right)+(-1)^{a} p F_{k, r-a}-p^{2} F_{k, r}\right] \\
\text { If } & \mathrm{a}=1 \text { and r=0, then } p_{k, j}^{j}=\frac{1}{1+k p-p^{2}}\left(\frac{1}{p^{n}}\left(p F_{k, n+1}+F_{k, n}\right)-p\right)
\end{aligned}
$$

Next we will apply this formula to the k-Lucas numbers. Because $L_{k, n}=\sigma_{1}^{n}+\sigma_{2}^{n}$, we can prove of a similar form

\subsection{Corollary}

$\sum_{j=0}^{n} \frac{L_{k, a j+r}}{p^{j}}=\frac{1}{p L_{k, a}-(-1)^{a}-p^{2}}\left[\frac{1}{p^{n}}\left(p L_{k, a(n+1)+r}-(-1)^{a} L_{k, a n+r}\right)+(-1)^{a} p L_{k, r-a}-p^{2} L_{k, r}\right]$

If $\mathrm{a}=1$ and $\mathrm{r}=0$, then

$$
\sum_{j=0}^{n} \frac{L_{k, j}}{p^{j}}=\frac{1}{1+k p-p^{2}}\left(\frac{1}{p^{n}}\left(p L_{k, n+1}+L_{k, n}\right)+k p-2 p^{2}\right)
$$

\subsection{Particular cases}

From Equation (4) and Equation (5),

a) $\quad p=k: \sum_{j=0}^{n} \frac{F_{k, j}}{k^{j}}=\frac{F_{k, n+2}}{k^{n}}-k \rightarrow \sum_{j=0}^{n} F_{j}=F_{n+2}-1$

$$
\sum_{j=0}^{n} \frac{L_{k, j}}{k^{j}}=\frac{L_{k, n+2}}{k^{n}}-k^{2} \rightarrow \sum_{j=0}^{n} L_{j}=L_{n+2}-1
$$

b) $\quad p=\frac{1}{2}: \sum_{j=0}^{n} 2^{j} F_{k, j}=\frac{1}{2 k+3}\left[2^{n+1}\left(F_{k, n+1}+2 F_{k, n}\right)-2\right] \rightarrow \sum_{j=0}^{n} 2^{j} F_{j}=\frac{1}{5}\left(2^{n+1} L_{n+1}-2\right)$

$$
\sum_{j=0}^{n} 2^{j} L_{k, j}=\frac{1}{2 k+3}\left[2^{n+1}\left(L_{k, n+1}+2 L_{k, n}\right)+2 k-2\right] \rightarrow \sum_{j=0}^{n} 2^{j} L_{j}=2^{n+1} F_{n+1}[1]
$$

c) For $\mathrm{p}=1$ we obtain the classical formulas

$$
\begin{aligned}
& \sum_{j=0}^{n} F_{k, j}=\frac{1}{k}\left(F_{k, n+1}+F_{k, n}-1\right) \rightarrow \sum_{j=0}^{n} F_{j}=F_{n+2}-1 \\
& \sum_{j=0}^{n} L_{k, j}=\frac{1}{k}\left(L_{k, n+1}+L_{k, n}+k-2\right) \rightarrow \sum_{j=0}^{n} L_{j}=L_{n+2}-1
\end{aligned}
$$




\subsection{Corollary (Forthe alternated sums)}

If $p=-r$, the equations (4) and (5) become

$$
\begin{aligned}
& \sum_{j=0}^{n}(-1)^{j} \frac{F_{k, j}}{r^{j}}=\frac{1}{1-k r-r^{2}}\left(\frac{(-1)^{n}}{r^{n}}\left(-r F_{k, n+1}+F_{k, n}\right)+r\right) \\
& \sum_{j=0}^{n}(-1)^{j} \frac{L_{k, j}}{r^{j}}=\frac{1}{1-k r-r^{2}}\left(\frac{(-1)^{n}}{r^{n}}\left(-r L_{k, n+1}+L_{k, n}\right)-r k-2 r^{2}\right)
\end{aligned}
$$

Then

a) If $r=1: \sum_{j=0}^{n}(-1)^{j} F_{k, j}=\frac{1}{k}\left((-1)^{n}\left(F_{k, n+1}-F_{k, n}\right)-1\right) \rightarrow \sum_{j=0}^{n}(-1)^{j} F_{j}=(-1)^{n} F_{n-1}-1$

$$
\sum_{j=0}^{n}(-1)^{j} l_{k, j}=\frac{1}{k}\left((-1)^{n}\left(L_{k, n+1}-L_{k, n}\right)+k+2\right) \rightarrow \sum_{j=0}^{n}(-1)^{j} L_{j}=(-1)^{n} L_{n-1}+3
$$

b) If $r=2: \sum_{j=0}^{n}(-1)^{j} \frac{F_{k, j}}{2^{j}}=\frac{1}{2 k+3}\left(\frac{(-1)^{n}}{2^{n}}\left(2 F_{k, n+1}-F_{k, n}\right)-2\right) \rightarrow \sum_{j=0}^{n}(-1)^{j} \frac{F_{j}}{2^{j}}=\frac{1}{5}\left((-1)^{n} \frac{L_{n}}{2^{n}}-2\right)$

$$
\sum_{j=0}^{n}(-1)^{j} \frac{L_{k, j}}{2^{j}}=\frac{1}{2 k+3}\left(\frac{(-1)^{n}}{2^{n}}\left(2 L_{k, n+1}-L_{k, n}\right)+2 k+8\right) \rightarrow \sum_{j=0}^{n}(-1)^{j} \frac{L_{j}}{2^{j}}=(-1)^{n} \frac{F_{n}}{2^{n}}+2
$$

c) If $r=k: \sum_{j=0}^{n}(-1)^{j} \frac{F_{k, j}}{k^{j}}=\frac{1}{2 k^{2}-1}\left(\frac{(-1)^{n}}{k^{n}}\left(k F_{k, n+1}-F_{k, n}\right)-k\right)$

$$
\sum_{j=0}^{n}(-1)^{j} \frac{L_{k, j}}{k^{j}}=\frac{1}{2 k^{2}-1}\left(\frac{(-1)^{n}}{k^{n}}\left(k L_{k, n+1}-L_{k, n}\right)+3 k^{2}\right)
$$

d) If $r=\frac{1}{p}: \sum_{j=0}^{n}(-1)^{j} p^{j} F_{k, j}=\frac{1}{p^{2}-k p-1}\left((-1)^{n} p^{n+1}\left(p F_{k, n}-F_{k, n+1}\right)+p\right)$

$$
\sum_{j=0}^{n}(-1)^{j} p^{j} L_{k, j}=\frac{1}{p^{2}-k p-1}\left((-1)^{n} p^{n+1}\left(p L_{k, n}-L_{k, n+1}\right)-k p-2\right)
$$

\subsection{Corollary}

By summing up the formulas (5) and (4),

$\sum_{j=0}^{n} \frac{L_{k, j}+F_{k, j}}{p^{j}}=\frac{1}{p^{n}\left(1+k p-p^{2}\right)}\left((p k+p+2) F_{k, n+1}+(2 p+1-k)\left(F_{k, n}-p^{n+1}\right)\right)$

Proof. Taking into account $L_{k, n}=F_{k, n+1}+F_{k, n-1}=2 F_{k, n+1}-k F_{k, n}$, it is

$\sum_{j=0}^{n} \frac{L_{k, j}+F_{k, j}}{p^{j}}=2 \sum_{j=0}^{n} \frac{F_{k, j+1}}{p^{j}}+(1-k) \sum_{j=0}^{n} \frac{F_{k, j}}{p^{j}}$

On the auther hand, $\sum_{j=0}^{n} \frac{F_{k, j+1}}{p^{j}}=\frac{1}{1+k p-p^{2}}\left(\frac{1}{p^{n}}\left(p F_{k, n+2}+F_{k, n+1}\right)-p^{2}\right)$ because $\sum_{j=0}^{n} \frac{F_{k, j+1}}{p^{j}}=p \sum_{j=0}^{n} \frac{F_{k, j}}{p^{j}}+\frac{F_{k, n+1}}{p^{n}}$ and then we apply Formula (4). So,

$\sum_{j=0}^{n} \frac{L_{k, j}+F_{k, j}}{p^{j}}=2 \sum_{j=0}^{n} \frac{F_{k, j+1}}{p^{j}}+(1-k) \sum_{j=0}^{n} \frac{F_{k, j}}{p^{j}}=\frac{2}{1+k p-p^{2}}\left(\frac{1}{p^{n}}\left(p F_{k, n+2}+F_{k, n+1}\right)-p^{2}\right)+\frac{1-k}{1+k p-p^{2}}\left(\frac{1}{p^{n}}\left(p F_{k, n+1}+F_{k, n}\right)-p\right)$

$=\frac{1}{p^{n}\left(1+k p-p^{2}\right)}\left(2 p F_{k, n+2}+2 F_{k, n+1}+p F_{k, n+1}+F_{k, n}-k p F_{k, n+1}-k F_{k, n}\right)-\frac{2 p^{2}+p-p k}{1+k p-p^{2}}$

$=\frac{1}{p^{n}\left(1+k p-p^{2}\right)}\left((p k+p+2) F_{k, n+1}+(2 p+1-k)\left(F_{k, n}-p^{n+1}\right)\right)$ 


\section{BINOMIAL TRANSFORM}

In this section we study the binomial transform of some of the previous numbers. We find also the main theorem of this paper.

\subsection{Theorem: First binomial transform.}

$\sum_{j=0}^{n}\left(\begin{array}{c}n \\ j\end{array}\right) k^{j} F_{k, a n+r+j}=F_{k,(a+2) n+r}$

Proof.Taking into account $1+k \sigma=\sigma^{2}$ it is

$\sum_{j=0}^{n}\left(\begin{array}{c}n \\ j\end{array}\right) k^{j} F_{k, a n+r+j}=\frac{1}{\sigma_{1}-\sigma_{2}} \sum_{j=0}^{n}\left(\begin{array}{c}n \\ j\end{array}\right)\left(\sigma_{1}^{a n+r}\left(k \sigma_{1}\right)^{j}-\sigma_{2}^{a n+r}\left(k \sigma_{2}\right)^{j}\right)=\frac{1}{\sigma_{1}-\sigma_{2}}\left(\sigma_{1}^{a n+r}\left(1+k \sigma_{1}\right)^{n}-\sigma_{2}^{a n+r}\left(1+k \sigma_{1}\right)^{n}\right)$

$=\frac{1}{\sigma_{1}-\sigma_{2}}\left(\sigma_{1}^{a n+r} \sigma_{1}^{2 n}-\sigma_{2}^{a n+r} \sigma_{2}^{2 n}\right)=F_{k,(a+2) n+r}$

In similar form, $\sum_{j=0}^{n}\left(\begin{array}{c}n \\ j\end{array}\right) k^{j} L_{k, a n+r+j}=L_{k,(a+2) n+r}$

\subsection{Theorem (Second binomial transform: Alternated binomial sums with the k- Fibonacci numbers)}

Binomial transform.

$\sum_{j=0}^{n}(-1)^{j}\left(\begin{array}{c}n \\ j\end{array}\right) \frac{F_{k, a n+r+j}}{k^{j}}=(-1)^{n} \frac{F_{k,(a-1) n+r}}{k^{n}}(8)$

Proof. Taking into account $\sigma_{1}=-\frac{1}{\sigma_{2}}$ and $1+k \sigma=\sigma^{2}$, it is

$$
\begin{aligned}
& \sum_{j=0}^{n}(-1)^{j}\left(\begin{array}{l}
n \\
j
\end{array}\right) \frac{F_{k, a n+r+j}}{k^{j}}=\frac{1}{\sigma_{1}-\sigma_{2}} \sum_{j=0}^{n}\left(\begin{array}{c}
n \\
j
\end{array}\right)\left(\sigma_{1}^{a n+r}\left(\frac{-\sigma_{1}}{k}\right)^{j}-\sigma_{2}^{a n+r}\left(\frac{-\sigma_{2}}{k}\right)^{j}\right)=\frac{1}{\sigma_{1}-\sigma_{2}}\left(\sigma_{1}^{a n+r}\left(1-\frac{\sigma_{1}}{k}\right)^{n}-\sigma_{2}^{a n+r}\left(1-\frac{\sigma_{2}}{k}\right)^{n}\right) \\
& =\frac{1}{\sigma_{1}-\sigma_{2}}\left(\sigma_{1}^{a n+r}\left(\frac{-1}{k \sigma_{1}}\right)^{n}-\sigma_{2}^{a n+r}\left(\frac{-1}{k \sigma_{2}}\right)^{n}\right)=\frac{1}{\sigma_{1}-\sigma_{2}} \frac{(-1)^{n}}{k^{n}}\left(\sigma_{1}^{a n+r-n}-\sigma_{2}^{a n+r-n}\right)=\frac{(-1)^{n}}{k^{n}} F_{k,(a-1) n+r}
\end{aligned}
$$

\subsection{Corollary}

$\sum_{j=0}^{n}(-1)^{j}\left(\begin{array}{l}n \\ j\end{array}\right) \frac{L_{k, a n+r+j}}{k^{j}}=(-1)^{n} \frac{L_{k,(a-1) n+r}}{k^{n}}$

The proof is similar to the previous.

The following formula is an adaptation of the Formula (1.6) in [2]

\section{MAIN THEOREM}

Let $t$ be an indeterminate. Then the following identity holds for $n=1,2,3, \ldots$

$\sum_{j=0}^{n}\left(\frac{t}{k}\right)^{j}\left(t F_{k, j-r+2}-k F_{k, j-r+1}\right)=\left(\frac{t}{k}\right)^{n} t F_{k, n-r+2}-k F_{k,-r+1}$

Proof. First, $1+(t-1) \sigma^{2}=1+t \sigma^{2}-\sigma^{2}=t \sigma^{2}-k \sigma=\sigma(t \sigma-k)$, and applying the Binet identity

$\sum_{j=0}^{n}\left(\frac{t}{k}\right)^{j}\left(t F_{k, j-r+2}-k F_{k, j-r+1}\right)=\frac{1}{\sigma_{1}-\sigma_{2}} \sum_{j=0}^{n}\left(\frac{t}{k}\right)^{j}\left(t\left(\sigma_{1}^{j-r+2}-\sigma_{2}^{j-r+2}\right)-k\left(\sigma_{1}^{j-r+1}-\sigma_{2}^{j-r+1}\right)\right)$ 


$$
\left.\begin{array}{l}
=\frac{1}{\sigma_{1}-\sigma_{2}}\left[\sigma_{1}^{-r+1}\left(t \sigma_{1}-k\right) \sum_{j=0}^{n}\left(\frac{t \sigma_{1}}{k}\right)^{j}-\sigma_{2}^{-r+1}\left(t \sigma_{2}-k\right) \sum_{j=0}^{n}\left(\frac{t \sigma_{2}}{k}\right)^{j}\right] \\
=\frac{1}{\sigma_{1}-\sigma_{2}}\left[\sigma_{1}^{-r+1}\left(t \sigma_{1}-k\right) \frac{\left(\frac{t \sigma_{1}}{k}\right)^{n+1}-1}{\frac{t \sigma_{1}}{k}-1}-\sigma_{2}^{-r+1}\left(t \sigma_{2}-k\right) \frac{\left.\left(\frac{t \sigma_{2}}{k}\right)^{n+1}-1\right]}{\frac{t \sigma_{2}}{k}-1}\right] \\
=\frac{k}{\sigma_{1}-\sigma_{2}}\left[\sigma_{1}^{-r+1} \frac{\left(t \sigma_{1}\right)^{n+1}-k^{n+1}}{\left.k^{n+1}-\sigma_{2}^{-r+1} \frac{\left(t \sigma_{2}\right)^{n+1}-k^{n+1}}{k^{n+1}}\right]}\right. \\
=\frac{k}{\sigma_{1}-\sigma_{2}}\left[\left(\frac{t}{k}\right)^{n+1} \sigma_{1}^{n-r+2}-\sigma_{1}^{-r+1}-\left(\frac{t}{k}\right)^{n+1} \sigma_{2}^{n-r+2}+\sigma_{2}^{-r+1}\right]=\left(\frac{t}{k}\right)^{n} t F_{k, n-r+2}-k F_{-r+1} \\
=\frac{k}{\sigma_{1}-\sigma_{2}}\left[\left(\frac{t}{k}\right)^{n+1} \sigma_{1}^{n-r+2}-\sigma_{1}^{-r+1}-\left(\frac{t}{k}\right)^{n+1} \sigma_{2}^{n-r+2}+\sigma_{2}^{-r+1}\right]=\left(\frac{t}{k}\right)^{n} t F_{k, n-r+2}-k F_{k,-r+1} \\
=\frac{1}{\sigma_{1}-\sigma_{2}}\left[\frac{t \sigma_{1}}{k}\right)^{n+1}-1 \\
\frac{t \sigma_{1}}{k}-1
\end{array}\right]
$$

Taking into account $L_{k, n}=F_{k, n-1}+F_{k, n+1}$, this formula can be written as

$\sum_{j=0}^{n}\left(\frac{t}{k}\right)^{j}\left(L_{k, j-r+1}+(t-2) F_{k, j-r+2}\right)=\left(\frac{t}{k}\right)^{n} t F_{k, n-r+2}-k F_{k,-r+1}$

\section{Particular cases:}

- $\quad t=k^{2} \rightarrow \sum_{j=0}^{n} k^{j}\left(k F_{k, j-r+2}-F_{k, j-r+1}\right)=k^{n+1} F_{k, n-r+2}-F_{k,-r+1}$

- $\quad r=1 \rightarrow \sum_{j=0}^{n}\left(\frac{t}{k}\right)^{j}\left(t F_{k, j+1}-k F_{k, j}\right)=\left(\frac{t}{k}\right)^{n} t F_{k, n+1}$

- $\quad k=r=1 \rightarrow \sum_{j=0}^{n} t^{j}\left(t F_{j+1}-F_{j}\right)=t^{n+1} F_{n+1}$

- $\quad r=0 \rightarrow \sum_{j=0}^{n}\left(\frac{t}{k}\right)^{j}\left(t F_{k, j+2}-k F_{k, j+1}\right)=\left(\frac{t}{k}\right)^{n} t F_{k, n+2}-k$

Is similar form we can prove the following corollary.

\subsection{Corollary. Formula (9) for the k-Lucas numbers.}

And then

$$
\sum_{j=0}^{n}\left(\frac{t}{k}\right)^{j}\left(t L_{k, j-r+2}-k L_{k, j-r+1}\right)=\left(\frac{t}{k}\right)^{n} t L_{k, n-r+2}-k L_{k,-r+1}
$$

$$
\text { - } \quad r=1 \rightarrow \sum_{j=0}^{n}\left(\frac{t}{k}\right)^{j}\left(t L_{k, j+1}-k L_{k, j}\right)=\left(\frac{t}{k}\right)^{n} t L_{k, n+1}-2 k
$$


- $\quad r=0 \rightarrow \sum_{j=0}^{n}\left(\frac{t}{k}\right)^{j}\left(t L_{k, j+2}-k L_{k, j+1}\right)=\left(\frac{t}{k}\right)^{n} t L_{k, n+2}-k^{2}$

\subsection{Theorem. Formula (9) for the alternated sums.}

$$
\begin{aligned}
& \sum_{j=0}^{n}(-1)^{j}\left(\frac{t}{k}\right)^{j}\left(t F_{k, j-r+2}-k F_{k, j-r+1}\right) \\
& =\frac{k}{t^{2}-k t-k^{2}}\left[(-1)^{n}\left(\frac{t}{k}\right)^{n+1}\left(\left(t^{2}+k^{2}\right) F_{k, n-r+2}-k t L_{k, n-r+2}\right)+\left(t^{2}+k^{2}\right) F_{k,-r+1}-k t L_{k,-r+1}\right]
\end{aligned}
$$

To prove this theorem we must apply the following identities:

$$
\begin{aligned}
& \sigma_{1} \cdot \sigma_{2}=-1, \quad \sigma_{1}+\sigma_{2}=k, \quad \sigma_{1}-\sigma_{2}=\sqrt{k^{2}+4}, \quad 1+(t-1) \sigma^{2}=\sigma(t \sigma-k), \quad\left(t \sigma_{1}+k\right)\left(t \sigma_{2}+k\right)=-\left(t^{2}-k^{2} t-k^{2}\right), \\
& \left(t \sigma_{1}-k\right)\left(t \sigma_{2}+k\right)=-\left(t^{2}-k t \sqrt{k^{2}+4}-k^{2}\right), \quad\left(t \sigma_{1}+k\right)\left(t \sigma_{2}-k\right)=-\left(t^{2}+k t \sqrt{k^{2}+4}-k^{2}\right)
\end{aligned}
$$

Proof.

$$
\begin{aligned}
\sum_{j=0}^{n}(-1)^{j} & \left(\frac{t}{k}\right)^{j}\left(t F_{k, j-r+2}-k F_{k, j-r+1}\right)=\frac{1}{\sigma_{1}-\sigma_{2}} \sum_{j=0}^{n}\left(\frac{-t}{k}\right)^{j}\left(t\left(\sigma_{1}^{j-r+2}-\sigma_{2}^{j-r+2}\right)-k\left(\sigma_{1}^{j-r+1}-\sigma_{2}^{j-r+1}\right)\right) \\
= & \frac{1}{\sigma_{1}-\sigma_{2}} \sum_{j=0}^{n}\left(\frac{-t}{k}\right)^{j}\left(t\left(\sigma_{1}^{j-r+2}-\sigma_{2}^{j-r+2}\right)-k\left(\sigma_{1}^{j-r+1}-\sigma_{2}^{j-r+1}\right)\right) \\
= & \frac{1}{\sigma_{1}-\sigma_{2}} \sum_{j=0}^{n}\left(\left(\frac{-t \sigma_{1}}{k}\right)^{j} \sigma_{1}^{-r+1}\left(t \sigma_{1}-k\right)-\left(\frac{-t \sigma_{2}}{k}\right)^{j} \sigma_{2}^{-r+1}\left(t \sigma_{2}-k\right)\right) \\
= & \frac{1}{\sigma_{1}-\sigma_{2}}\left(\sigma_{1}^{-r+1}\left(t \sigma_{1}-k\right) \frac{\left(\frac{-t \sigma_{1}}{k}\right)^{n+1}-1}{\frac{-t \sigma_{1}}{k}-1}-\sigma_{2}^{-r+1}\left(t \sigma_{2}-k\right) \frac{\left(\frac{-t \sigma_{2}}{k}\right)^{n+1}-1}{\frac{-t \sigma 2}{k}-1}\right) \\
= & \frac{-k}{\sigma_{1}-\sigma_{2}}\left(\frac{t \sigma_{1}-k}{t \sigma_{1}+k}\left(\left(\frac{-t}{k}\right)^{n+1} \sigma_{1}^{n-r+2}-\sigma_{1}^{-r+1}\right)-\frac{t \sigma_{2}-k}{t \sigma_{2}+k}\left(\left(\frac{-t}{k}\right)^{n+1} \sigma_{2}^{n-r+2}-\sigma_{2}^{-r+1}\right)\right) \\
= & \frac{-k}{\sigma_{1}-\sigma_{2}}\left(\left(\frac{-t}{k}\right)^{n+1}\left(\frac{t \sigma_{1}-k}{t \sigma_{1}+k} \sigma_{1}^{n-r+2}-\frac{t \sigma_{2}-k}{t \sigma_{2}+k} \sigma_{1}^{n-r+2}\right)-\left(\frac{t \sigma_{1}-k}{t \sigma_{1}+k} \sigma_{1}^{-r+1}-\frac{t \sigma_{2}-k}{t \sigma_{2}+k} \sigma_{2}^{-r+1}\right)\right) \\
= & \frac{-k}{-t^{2}+k^{2} t+k^{2}} \frac{1}{\sigma_{1}-\sigma_{2}}\left(\left(\frac{-t}{k}\right)^{n+1}\left(\left(-t^{2}+k t \sqrt{k^{2}+4}-k^{2}\right) \sigma_{1}^{n-r+2}+\left(t^{2}+k t \sqrt{k^{2}+4}+k^{2}\right) \sigma_{2}^{n-r+2}\right)\right) \\
- & \frac{-k}{-t^{2}+k^{2} t+k^{2}} \frac{1}{\sigma_{1}-\sigma_{2}}\left(\left(-t^{2}+k t \sqrt{k^{2}+4}-k^{2}\right) \sigma_{1}^{-r+1}+\left(t^{2}+k t \sqrt{k^{2}+4}+k^{2}\right) \sigma_{2}^{-r+1}\right) \\
= & \frac{-k}{-t^{2}+k^{2} t+k^{2}}\left(\frac{t}{k}\right)^{n+1}\left(\frac{\left(-t^{2}-k^{2}\right) \sigma_{1}^{n-r+2}+\left(t^{2}+k^{2}\right) \sigma_{2}^{n-r+2}}{\sigma_{1}-\sigma_{2}}+k t \sqrt{k^{2}+4} \frac{\sigma_{1}^{n-r+2}+\sigma_{2}^{n-r+2}}{\sqrt{k^{2}+4}}\right) \\
& +\frac{-k}{-t^{2}+k^{2} t+k^{2}}\left(\frac{\left(-t^{2}-k^{2}\right) \sigma_{1}^{-r+1}+\left(t^{2}+k^{2}\right) \sigma_{2}^{-r+1}}{\sigma_{1}-\sigma_{2}}+k t \sqrt{k^{2}+4} \frac{\sigma_{1}^{-r+1}+\sigma_{2}^{-r+1}}{\sqrt{k^{2}+4}}\right) \\
= & \frac{k}{-t^{2}+k^{2} t+k^{2}}\left((-1)^{n+1}\left(\frac{t}{k}\right)^{n-r+1}\left(\left(t^{2}+k^{2}\right) F_{k, n-r+2}-k t L_{k, n-r+2}\right)-\left(\frac{t}{k}\right)^{-r}\left(\left(t^{2}+k^{2}\right) F_{k,-r+1}-k t L_{k,-r+1}\right)\right) \\
= & \frac{k}{t^{2}-k^{2} t-k^{2}}\left((-1)^{n}\left(\frac{t}{k}\right)^{n+1}\left(\left(t^{2}+k^{2}\right) F_{k, n-r+2}-k t L_{k, n-r+2}\right)+\left(t^{2}+k^{2}\right) F_{k,-r+1}-k t L_{k,-r+1}\right) \\
& \\
&
\end{aligned}
$$




$$
\begin{aligned}
& r=1: \sum_{j=0}^{n}(-1)^{j}\left(\frac{t}{k}\right)^{j}\left(t F_{k, j+1}-k F_{k, j}\right)=\frac{k}{t^{2}-k^{2} t-k^{2}}\left[(-1)^{n}\left(\frac{t}{k}\right)^{n+1}\left(\left(t^{2}+k^{2}\right) F_{k, n+1}-k t L_{k, n+1}\right)-2 k t\right] \\
& t=k: \sum_{j=0}^{n}(-1)^{j} k\left((k-1) F_{k, j-r+1}+F_{k, j-r}\right)=(-1)^{n}\left(\left(k^{2}-2 k+2\right) F_{k, n-r+1}+(k-2) F_{k, n-r}\right)+(-1)^{r}\left((k-2) F_{k, r-1}-2 F_{k, r}\right)
\end{aligned}
$$

If $t=k=1$, for the classical Fibonacci numbers it is $\sum_{j=0}^{n}(-1)^{j} F_{j-r}=(-1)^{n} F_{n-r-1}-(-1)^{r} F_{r+2}$

If $t=k=2$, for the Pell numbers it is $\sum_{j=0}^{n}(-1)^{j}\left(P_{j-r+1}+P_{j-r}\right)=(-1)^{n} P_{n-r+1}-(-1)^{r} P_{r}$

Similarly, for the alternated sum with k-Lucas numbers, one can prove this last corollary.

\subsection{Corolllary.}

$$
\begin{aligned}
& \sum_{j=0}^{n}(-1)^{j}\left(\frac{t}{k}\right)^{j}\left(t L_{k, j-r+2}-k L_{k, j-r+1}\right) \\
& \quad=\frac{k}{t^{2}-k t-k^{2}}\left[(-1)^{n}\left(\frac{t}{k}\right)^{n+1}\left(\left(t^{2}+k^{2}\right) L_{k, n-r+2}-k t\left(k^{2}+4\right) F_{k, n-r+2}\right)+\left(t^{2}+k^{2}\right) L_{k,-r+1}-k t\left(t^{2}+4\right) F_{k,-r+1}\right]
\end{aligned}
$$

\section{REFERENCES}

1. Bhatnagar, G. 2016. Analoques of a Fibonacci-Lucas identity. The Fibonacci Quarterly, 54(2), 166-171.

2. Falcon, S. 2001. On the k-Lucas Numbers. International Journal of Contemporary Mathematical Sciences, 6(21).2011, 1039-1050.

3. Falcon, S. and Plaza, A. 2007. On the Fibonacci k-numbers. Chaos, Solitons \& Fractals, 32(5),1615-1624.

4. Horadam, A. F. 1961. A generalized Fibonacci sequence. Math Mag, 68, 455-459.

5. El Naschie, M.S. 2009. E-eight exceptional Lie groups, Fibonacci lattices and the standard model. Chaos, Solit.\& Fract.41(3), 1340-1343. 